\title{
Post-breakdown Transient Characteristics of a Gas-Filled Plasma Closing Switch
}

\author{
Yuan Yao, Igor Timoshkin, Senior Member, IEEE, S. J. MacGregor, Senior Member, IEEE, M. P. \\ Wilson, Member, IEEE, M. J. Given, Senior Member, IEEE, T. Wang
}

\begin{abstract}
Gas-filled plasma closing switches (PCSs) are essential components of high energy density pulsed power systems, used to generate short, high-power (10s MW to several GW) impulses. In practical applications, PCSs are required to operate in high current $(10 \mathrm{~s} \mathrm{kA})$, high voltage $(10 \mathrm{~s}-100 \mathrm{~s} \mathrm{kV})$ regimes, to produce fast-rising ns and sub-ns $\mathrm{HV}$ impulses. The transient impedance of a PCS affects the rise time of the generated impulses and the power delivered to the load. Thus, design and optimisation of PCSs require detailed information on the dynamic plasma resistance.

The present paper is focused on an investigation of the timedependent resistance of the plasma channel(s) formed between the electrodes of a multi-electrode PCS. Experimentally-measured underdamped current and voltage waveforms were used to obtain the transient plasma resistance of the PCS. The PCS was filled with different gases: dry air, $\mathrm{CO}_{2}, \mathrm{~N}_{2}$ and a $90 \% / 10 \% \mathrm{Ar} / \mathrm{O}_{2}$ mixture, in the pressure range from 0 bar up to 10 bar (gauge). It was found that the plasma resistance drops rapidly from a few hundreds of Ohms to a few hundreds of milli-Ohms due to the Joule heating of the breakdown channel. The methods proposed by Braginskii and Kushner to model the transient post-breakdown resistance were used to obtain analytical plasma resistances for all of the gasses. These resistances have been compared with the transient resistance obtained using a Kirchhoff analysis of the lumped-element, post-breakdown $R L C$ circuit. Based on this comparison, the suitability of the chosen analytical methods to determine the transient breakdown resistance in gases is discussed. The obtained results will help in optimisation of the operational performance of PCSs filled with environmentallyfriendly, low environmental impact gases.
\end{abstract}

Index Terms - Plasma closing switch; plasma channel; transient plasma resistance

\section{INTRODUCTION}

$\mathrm{T}$ ransient spark discharges in gases have found multiple practical applications in modern power and pulsed power high voltage systems. In particular, gas-filled plasma closing switches (PCS), one of the critical components of many practical pulsed power systems, are based on gas breakdown, and the formation of a conductive plasma channel between the electrodes of the switch. PCSs are employed in different pulsed power systems and machines, such as linear transformer drivers

Y. Yao, I. Timoshkin, S. J. MacGregor, M. P. Wilson, M. J. Given, T. Wang are with the Department of Electronic and Electrical Engineering, University of Strathclyde, Glasgow, G1 1XW, UK, (e-mail: yuan.yao.101@strath.ac.uk).
(LTDs), [1], Marx generators for Z-pinches, [2], apparatuses for the generation of ultra-wide band electromagnetic impulses, [3], and generators of high voltage impulses for biomedical and environmental applications, [4]. Over the past several decades, a significant variety of different topologies of gas-filled PCSs has been developed, and their operational characteristics have been investigated and reported, [5]. However, there is still a need for further characterisation of the operational performance of PCSs, which will assist in the design and optimisation of pulsed power systems. One such parameter that requires further characterisation and investigation, in order to realise optimal performance of pulsed power machines in specific practical applications, is the dynamic resistance of the spark/plasma channel developed in the gas-filled spark gap during the switching operation.

The dynamic resistance of the plasma channel may affect the rate of voltage collapse across the switch during its closure, [6]; the rate of energy deposition and the total energy deposited into the spark gap, the energy losses during the switching process, and electrode erosion, [7]. Typically, in switching operations, it is desirable to minimise the spark gap resistance, in order to increase the efficiency of the energy transfer, and to reduce the power loss in PCSs.

Also, in the case of fast switching operations, it is crucial to minimise the closure time of PCSs, [8], i.e. the time required for the plasma in the breakdown channel to become highly conductive. During this thermal process, the resistance of the plasma channel drops to its minimum value, which corresponds to the resistance of the conductive, post-breakdown plasma channel formed in the switch. Typically, in order to enable efficient operation of a pulsed power system, the transient time during which the resistance of the spark channel reduces to its minimum value should be as short as possible, and can be shorter than $\sim 1$ ns, [9].

Thus, the time-dependent, post-breakdown resistance is one of the important parameters that defines the operational performance of PCSs and thus underpins the operational characteristics of pulsed power systems in general.

The transient resistance of spark channels in gases has been investigated over many decades, starting with pioneering works by Toepler, [10], Rompe and Weizel, [11], and Braginskii, [12].

In Toepler's empirical model, the resistance of the plasma channel is directly proportional to the product of Toepler's coefficient and the length of the spark, and inversely proportional to the integral of the spark current. Toepler's coefficient is specific to each gas type, and can be obtained by fitting the calculated resistance to experimentally-measured values. 
Both the Rompe-Weizel and Braginskii models are based on the energy balance in the spark/plasma channel. In the RompeWeizel approach, it is assumed that the internal energy of the plasma is divided into two main components, the ionisation energy and the kinetic energy of electrons, with the latter equating to the energy delivered into the plasma channel through Joule heating. Thus, the dynamic resistance of the spark channel can be obtained, [11]. In the Braginskii approach, it is assumed that the energy delivered into the plasma channel through Joule heating is parted into the internal energy of the plasma and the work done by the expanding plasma channel, which has a constant internal pressure and drives a shock wave into the surrounding gas. Assuming that the pressure at the plasma-gas interface is proportional to the squared velocity of plasma channel expansion, the time-dependent radius and the resistance of the spark channel can be obtained.

Following these models, Kushner et al., [13], developed an analytical approach, assuming that the plasma in the spark channel is in local thermodynamic equilibrium, and the plasma conductivity is proportional to the plasma temperature raised to the power 3/2. Also, it was assumed that the plasma channel expands hydrodynamically, thus, if the time-dependent plasma channel radius is known, the dynamic resistance of the spark/plasma channel can be obtained.

Akiyama et. al, [14, 15], Engel et al., [16], and Greason, [17], also investigated the resistance of breakdown spark channels in different gases, and developed several methodologies to obtain the transient resistance of the spark discharge channel using experimental breakdown current and voltage waveforms. Despite these advances in the analysis of the transient resistance using the experimental waveforms, there were problems with derivation of the time-varying plasma channel resistance, attempted based on measurements of the voltage drop across the switch and the transient current through the switch, due to difficulties in separation of the resistive and inductive components of the switch impedance, [18].

Different approaches to this problem have been explored. In [25], the inductive voltage drop was obtained based on the estimated inductance of the switch used in this work. In [13], the time-dependent resistance of spark-gaps was obtained by using a microwave measurement technique, which requires information on the characteristic resistance of the transmission line and the inter-electrode capacitance. In [20], the postbreakdown circuit, including the plasma closing switch, was modelled as a lumped-element $R L C$ circuit, and the dynamic resistance was obtained based on a Kirchhoff analysis of this circuit. However, this approach requires detailed information on the circuit parameters, including inductance and, if applicable, the capacitance of the individual circuit elements. In many cases, it is problematic to obtain accurate values of these parameters and estimates have to be used. Nevertheless, this approach can provide a straightforward evaluation of the dynamic post-breakdown resistance. Therefore, in order to establish a reliable method of analysis of the plasma channel resistance in PCSs, the different models and approaches must be evaluated and compared. This analysis can help in further development and optimisation of advanced, gas-filled plasma closing switches for pulsed power applications.

In the present paper, a direct method and two analytical approaches to obtaining the post-breakdown plasma resistance have been used in analysis of the transient spark resistance.

The direct method of obtaining the transient plasma resistance is based on the $R L C$ lumped-element model of the post-breakdown circuit; this circuit model includes the elements of the pulse driving circuit and the plasma closing switch. By applying Kirchhoff's voltage law to this circuit, the equation for the transient post-breakdown resistance has been derived, and can be resolved from experimental current waveforms.

An analytical model that combines the hydrodynamic parameters of the spark channel at breakdown and the lumped circuit parameters of the pulse driving circuit has also been developed in the present study. This model takes into account the hydrodynamic radius of the expanding post-breakdown plasma channel based on the Braginskii approach [12], which is used to calculate the resistance of the post-breakdown plasma channel.

Another analytical approach used in the present paper is based on the phenomenological equation for the transient spark resistance at breakdown provided in [13] (Kushner's approach), which also requires information from the measured current waveform, and on the hydrodynamic radius of the spark channel.

All three methods were used to obtain the transient resistance of the plasma channel(s) in the PCS reported in [21], when filled with different gases: dry air, $\mathrm{CO}_{2}, \mathrm{~N}_{2}$ and a $90 \% / 10 \% \mathrm{Ar} / \mathrm{O}_{2}$ mixture, in the pressure range from 0 bar (gauge) up to 10 bar (gauge). Using the experimental datasets obtained and presented in [21], direct calculation of the time-dependent plasma resistance was conducted using Kirchhoff's approach. The plasma resistance values were also obtained using two analytical approaches: the Braginskii model, based on the transient radius of the plasma channel; and the modified Kushner approach, in which the analytical radius of the channel and the experimental breakdown current waveforms were used.

These approaches have mainly been used for modelling of the transient plasma resistance in the case of overdamped current oscillation in the circuit. However, in some practical cases, for optimisation of the operational characteristics of pulsed power switches, it is required to have detailed information on the dynamic behaviour of the plasma channel resistance in the case of underdamped current oscillation in the post-breakdown circuit. To address this issue, in the present paper, the applicability of both, Braginskii and Kushner, approaches to the underdamped transient process is investigated. The analytically-obtained dynamic plasma channel resistances were compared with the directly-obtained plasma channel resistances.

The analysis of the results produced by these methods allows for the evaluation of the applicability of the different models to obtain the transient post-breakdown resistance, and for the optimisation of the design and operational parameters of PCSs.

\section{EXPERIMENTAL SETUP}

The present study continues the work reported in [21], in which a multi-electrode plasma closing switch was designed and developed, and its self-breakdown characteristics were obtained for the gases and pressure range detailed in Section I. 
The main focus of the present paper is the investigation of the post-breakdown parameters of this PCS.

A schematic diagram of the high voltage experimental setup used is given in Fig. 1.

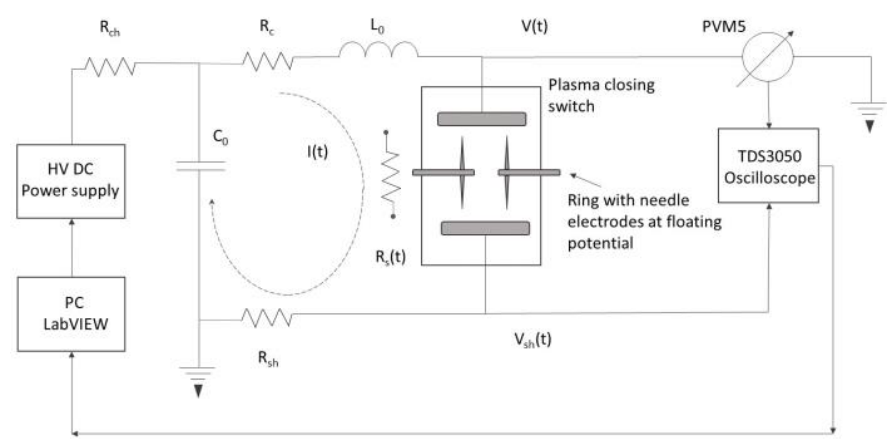

Fig. 1. Schematic diagram of the experimental setup.

An HV output of a Glassman high voltage de power supply was connected to a $1 \mathrm{M} \Omega$ charging resistor, $R_{c h}$. The voltage output from the dc power supply was controlled using LabVIEW code, via an RS-232 interface. This power supply was used to stress the upper plane electrode of the PCS, and to charge an $80 \mathrm{nF}$ capacitor, $C_{0}$, which was connected in parallel with the switch. The total inductance of the circuit is represented in Fig.1. by an inductor $L_{0}$. The post-breakdown circuit could be represented by a lumped $R L C$ circuit, the total resistance of the discharge circuit includes the time dependent resistance of the PCS, $R_{S}(t)$, and resistance $R_{0}$ which is the sum of the resistance of circuit components, $R_{c}$ and the resistance of the current shunt, $R_{s h}: R_{0}=R_{c}+R_{s h}$.

The plasma closing switch consists of two main, high voltage and ground, plane electrodes, and a central ring electrode, which has four sharp needles on each side. The central electrode of the switch with the corona discharge needles was at a floating potential. This plasma closing switch was described in detail in [21].

The breakdown voltage was obtained by using a NorthStar PVM-5 high voltage probe ( $80 \mathrm{MHz}$ bandwidth, $400 \mathrm{M} \Omega$ input resistance, and $12 \mathrm{pF}$ input capacitance) with 1000:1 division ratio. The PVM-5 probe was connected to the upper plane HV electrode and provided the voltage drop, $V(t)$, across the PCS, Fig.1. The transient current was obtained using a tubular lowinductance current shunt (Samtech Ltd, Scotland), $R_{s h}$, Fig.1. The voltage drop across the current shunt, $V_{s h}(t)$, was used to obtain the post-breakdown current in the circuit: $I(t)=V_{s h}(t) / R_{s h} . \quad$ All waveforms were acquired using a Tektronix TDS 3050 digitising oscilloscope $(500 \mathrm{MHz}$ bandwidth, $5 \mathrm{GS} / \mathrm{s}$ sampling rate).

The gas control system used in the present work to manage the gas environment in the PCS included a gas distribution board, a rotary vacuum pump to evacuate the switch prior to filling it with a fresh portion of gas, and a digital pressure controller, Alicat PC-1500PSIG-D, to set and control the pressure in the switch.

\section{ANALYTICAL MODELLING}

The transient pre-breakdown phase of the spark discharge process is completed by the formation of a conductive plasma channel between the energised and grounded electrodes. During the formation of the plasma channel, the resistance of the air gap between the electrodes drops significantly over a very short time, typically a few ns. After the formation of the plasma channel bridging the inter-electrode gap, a conductive current starts to flow through this channel resulting in its intensive Joule heating and thermalisation. Thus, the resistance of the plasma channel decreases rapidly during the first quarter-cycle of the underdamped current waveform, and reaches a minimum when the current through the channel is at its maximum. Therefore, the post-breakdown dynamic resistance of the plasma channel in the PCS is governed by the post-breakdown oscillation of the electrical charge in the circuit.

As discussed in Section I, there are several approaches aimed at modelling the dynamic behaviour of the post-breakdown resistance. The Toepler model, [10], is a phenomenological model which links the current in the post-breakdown circuit and the plasma resistance. The phenomenological nature of this approach limits its practical applications. The Rompe-Weizel [11] and Vlastós [22] models are based on the energy balance equation for the plasma channel, with a fixed plasma channel radius and varying plasma conductivity.

However, a comparison of transient spark resistances obtained experimentally and by different analytical methods, conducted in [16], demonstrated that the Braginskii and Kushner models of spark resistances provide the best fits between the experimental and analytical values.

The Braginskii model, [12], is based on the energy balance equation for the plasma channel, and assumes that the plasma conductivity in the channel remains constant during the transient post-breakdown process, with changes in resistance due only to the hydrodynamic expansion of the plasma channel. The time-dependent spark channel radius obtained by the Braginskii approach shows a good agreement with the experimental data, [23]. Kushner's model of the plasma channel resistance, [13], is based on the assumptions that: 1) the plasma conductivity is proportional to its temperature raised to the power $3 / 2$ (the Spitzer conductivity); 2) the channel expands hydrodynamically ("snowplow" model) and its initial crosssection is proportional to the degree of over-voltage raised to a specific power. This model was used to obtain the transient resistance of spark discharges in selected gases, including dry air, $\mathrm{Ar}, \mathrm{N}_{2}$ and $\mathrm{SF}_{6}$ [15].

In Section III, the Braginskii and Kushner approaches were applied to the underdamped transient process, and the analytically-obtained transient plasma channel resistances were compared with the resistances obtained using the direct method, based on the application of Kirchhoff's voltage law to the lumped-element model of the post-breakdown circuit.

\section{A. Braginskii Model for the Plasma Channel Radius}

The Braginskii model, [12], describes the dynamics of the expanding plasma channel during the energy deposition stage, when the conduction current flows through the plasma channel. This model assumes that, as current flows through the plasma channel, the Joule heating of the plasma results in a rapid 
increase in temperature and pressure within the channel. Thus, the channel expands and drives a strong shock wave into the surrounding gas.

The plasma channel expands and its radius increases (to simplify the notations, the time-dependent radius of the channel is given as $a$, i.e. $a \equiv a(t))$. In the Braginskii model, according to [10], $a$ is given by:

$$
a^{2}=\left(\frac{4}{\pi^{2} \rho_{0} \xi \sigma}\right)^{\frac{1}{3}} \int_{t 0}^{t} I(t)^{\frac{2}{3}} d t
$$

where $\sigma$ is the electrical conductivity of the plasma-filled channel, $\rho_{0}$ is the gas density (this model assumes that $\sigma$ and $\rho_{0}$ have constant values during the energy deposition stage); $\xi$ is a constant which depends on the gas properties - it is related to the effective ratio of specific heats, $\gamma$, assuming that the plasma in the breakdown channel can be considered to satisfy the ideal gas approximation. According to [25], $\gamma=1.25$ for common gases such as air and $\mathrm{N}_{2}$. An expression for $\xi$ was also obtained in [12]:

$$
\xi=K_{p}\left(1+\frac{1}{\gamma-1} \frac{1}{\dot{a}^{2}}\left(\ddot{a} a+\dot{a}^{2}\right)\right) \cong K_{p} \frac{\gamma}{\gamma-1}
$$

Braginskii assumed that $K_{p}=0.9$, thus, for air and nitrogen, $\xi=4.5$; in the case of $\mathrm{CO}_{2}$ and the $\mathrm{Ar} / \mathrm{O}_{2}$ mixture tested herein, $\xi$ values are 6.9 and 2.26 , respectively.

In the Braginskii approach, it is postulated that the conductivity of plasma is constant during the whole transient process. Although it is known that the plasma conductivity can vary during the transient current oscillation in the breakdown channel, it was shown in $[12,25]$ that these variations are not significant and the plasma conductivity used in (1) could be considered as being constant.

If the time varying radius, $a$, of the cylindrical plasma channel is known, the time-dependent resistance of this channel, $R_{s B}(t)$, obtained by the Braginskii approach, is given as:

$$
R_{S B}(t)=\frac{l}{\sigma \pi a^{2}}
$$

where $l$ is the length of the plasma channel. Inspection of (1) and (3) leads to the conclusion that at time moment, $t_{0}$, the plasma channel radius is $a_{0}$, and the plasma channel resistance is $R_{s B}\left(t_{0}\right)-t_{0}$ is the time at which the full conduction current starts to flow through the plasma channel. It was found in the present study that the initial value of $a_{0}$ has little effect on the plasma channel resistance at the later stage of the transient process. Thus, the behaviour of $R_{s B}(t)$ obtained by the Braginskii approach is not sensitive to the initial radius of the breakdown channel.

The conductivity of the plasma channel, $\sigma$, which is required for calculation of the plasma resistance, (3), can be obtained using the Spitzer model, which links the electronic density and the electrical conductivity of the plasma, [26]. The electronic densities in transient spark discharges in air, nitrogen, carbon dioxide and argon, in experimental conditions similar to those in the present paper, were obtained in [27], [28], [29] and [30], respectively. It was shown that the electronic density in such transient plasma discharges is $\sim\left(10^{22}-10^{23}\right) \mathrm{m}^{-3}$. Based upon these values of the electronic densities in the transient spark discharges, the plasma channel conductivity was estimated, and a nominal fixed value of $5000 \mathrm{~S} / \mathrm{m}$ was used in the calculations performed in the present work. Specific values of the electronic density and plasma conductivity for transient spark discharges developed in different gases, and using different electrical driving circuits, can be obtained using optical emission spectroscopy methods.

\section{B. The Kushner Model for Plasma Resistance}

The Kushner model for the dynamic resistance of the plasma breakdown channel, [16], assumes that the plasma in the breakdown channel is in local thermodynamic equilibrium during its expansion phase. This approach helps to overcome limitations of the Spitzer model, which postulates a high degree of ionisation in the spark channel. The Kushner equation for the time-dependent resistance of the plasma channel was obtained and discussed in $[16,31,32]$ :

$$
R_{S K}(t)=\beta K l\left(\frac{p^{3}}{A(t)^{2} I(t)^{6}}\right)^{\frac{1}{5}}
$$

where $l$ is the length of the plasma channel (in m); $p$ is the initial ambient gas pressure (in Pa); $A(t)=\pi a^{2}$ is the spark channel cross-sectional area (in $\mathrm{m}^{2}$ ), calculated using Braginskii's equation (1); $K$ is a constant; and the coefficient $\beta$ relates to field non-uniformity. For a point-plane topology, $\beta$ is defined as the ratio between the "average" field in the gap which can be calculated as "applied voltage divided by the gap distance" and the maximum field at the point electrode.

Values of $K$ were obtained in [16] and [31] for a quasiuniform electric field, and found to be $\sim 24.7$. However, as the electric field in the switch tested herein is highly divergent due to the presence of the needle electrodes, the coefficient $\beta$ was used in (4) to account for such field non-uniformity. In [32], it was found that $\beta$ is proportional to $E l / p$, where $E$ is the electrical field strength at breakdown. In [21], $\beta$ was derived for the inhomogeneous field in the PCS topology by field simulations and was found to be $\sim 1 / 11$. Thus, the product of $K$ and $\beta$ is $\sim 2.25$ for the present switch topology.

\section{The RLC Post-Breakdown Circuit Model}

When a final, breakdown plasma streamer bridges the interelectrode gap, the full conduction current starts to flow through this plasma channel, resulting in its fast Joule heating. The postbreakdown circuit, which includes the plasma channel and the pulse driving circuit, can be described using a lumped-element $R L C$ equivalent circuit. An equation for the time-dependent spark resistance, $R_{s}(t)$, can be obtained by resolving Kirchhoff's 
equation for this $R L C$ equivalent circuit. This equation for $R_{s}(t)$ is given as:

$$
\begin{gathered}
R_{S}(t)=\frac{1}{I(t)}\left\{V_{t h}-L_{0} \frac{d I(t)}{d t}\right. \\
\left.-\frac{1}{C_{0}} \int_{0}^{t} I(t) d t-R_{0} I(t)\right\}
\end{gathered}
$$

where $V_{t h}$ is the voltage across the plasma channel at the moment at which the plasma becomes thermalised; $I(t)$ is the transient current in the circuit; and $C_{0}, L_{0}$, and $R_{0}$ are the equivalent circuit capacitance, inductance and resistance, respectively, Fig.1.

In the present experimental system, the value of the capacitance, $C_{0}$ is $80 \mathrm{nF}$, and the total value of the circuit inductance, $L_{0}$, is $\sim 850 \mathrm{nH}$. This value of $L_{0}$ was calculated using analytical equations provided in [22], with topological parameters obtained from the experimental set-up.

The equivalent circuit resistance, $R_{0}$, was obtained using the transient current oscillation in the circuit with no PCS, i.e. in the short-circuit regime. After measuring the experimental short-circuit current waveform, $R_{0}$ was obtained by fitting an exponentially-decaying sinusoidal oscillation to the shortcircuit waveforms. Ten independent short-circuit current waveforms were used in this procedure, yielding $0.35 \Omega$ as an average value of $R_{0}$, with a standard deviation of $0.02 \Omega$.

The inductance of the spark channel was evaluated using an equation provided in [24], and it was found that this inductance (a few $\mathrm{nH}$ ) is significantly lower than $L_{0}$. Thus, the timedependent inductance of the spark channel was neglected in the present work. The capacitance of the plasma channel (the capacitance formed by the plasma channel and the grounded elements of the switch/pulsed power circuit, [33]) can also be neglected, as it is significantly lower than the capacitance of the energy storage capacitor in the pulse driving circuit, $C_{0}$.

\section{RESULTS}

\section{A. Breakdown Voltage and Post-Breakdown Current}

Breakdown voltage and post-breakdown current waveforms were obtained for the PCS, when filled with all investigated gases over the pressure range from 0 to $10 \mathrm{bar}$, in 1 bar increments. In each test, the breakdown voltage and current waveforms were obtained and recorded for 10 consecutive breakdown events, without changing the gas in the PCS. The typical waveforms for air at 6 bar are shown in Fig. 2.

When a breakdown occurs, the voltage collapses from the (pre-)breakdown value, $V_{b r}$, to its value across the thermal plasma channel, $V_{t h}$. At this moment, the conduction current starts to flow through the plasma, resulting in current and voltage oscillations, due to the underdamped behaviour of the $R L C$ circuit. The duration of the first quarter-cycle of the current oscillation (time to the first current peak) is $\sim 450 \mathrm{ns,}$ Fig. 2. It should be noted that $V_{b r}$ values were reported in [21] for all tested gases in the present PCS.

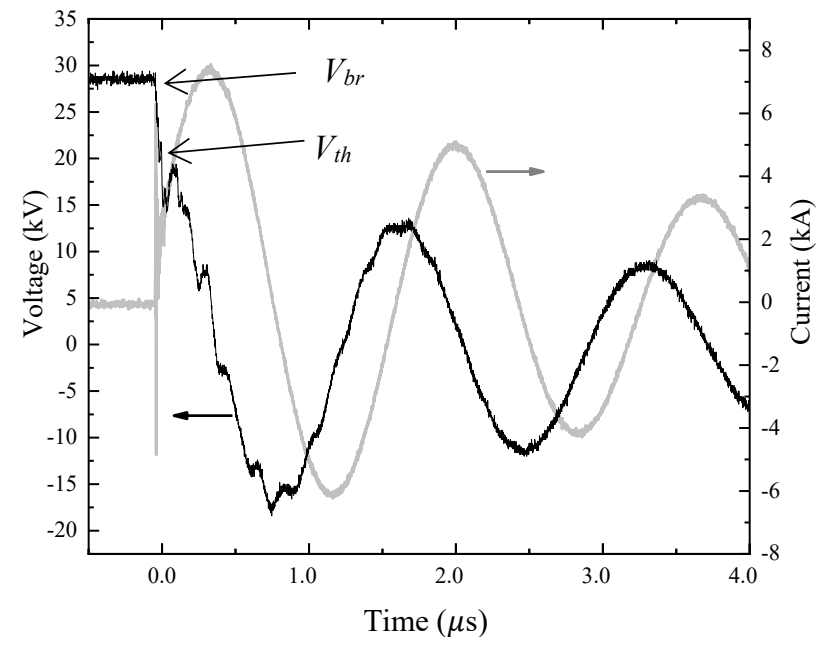

Fig. 2. The current and voltage waveforms of spark breakdown in air at 6 bar.

\section{B. Dynamic Radius of the Plasma Channel}

The time-dependent plasma channel radius was calculated by numerically solving (1), using the experimental postbreakdown current waveforms. Fig. 3 shows an example of the calculated plasma channel radius during the whole period of the current oscillation for dry air at 1 bar.

As can be seen from Fig. 3, the spark channel plasma radius increases rapidly during the first half-period of the current oscillation, and tends to increase with a slower rate after several current oscillations. This dynamic behaviour of the plasma channel formed in air is confirmed by the optical experimental observations reported in [23, 34].

The time-dependent plasma channel radius for all tested gases over the experimental pressure range has been calculated. At any particular gas pressure condition, 10 current waveforms were obtained and the data for these waveforms was averaged. This average waveform was then used in to calculate the time dependent radius using (3).

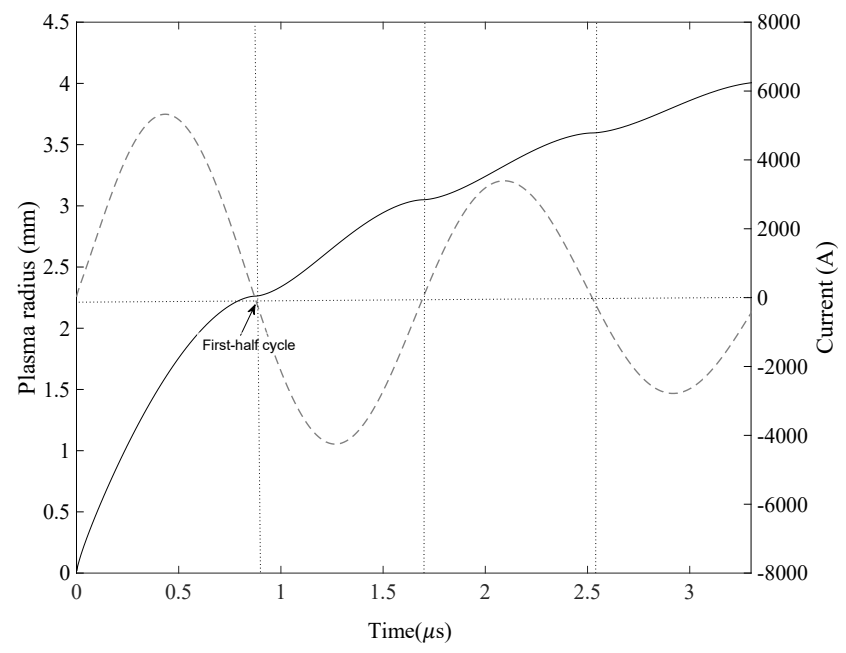

Fig. 3. The plasma channel radius- obtained by (3) for spark breakdown in dry air at 1 bar, solid line. Post-breakdown current wave-form is shown as a dashed 
line. Points of inflection in the plasma channel radius correspond to current zero events.

Examples of these radii for $\mathrm{N}_{2}$ and the $\mathrm{Ar} / \mathrm{O}_{2}$ mixture for time intervals shorter that the first half-cycle of the current oscillation are presented in Fig. 4.
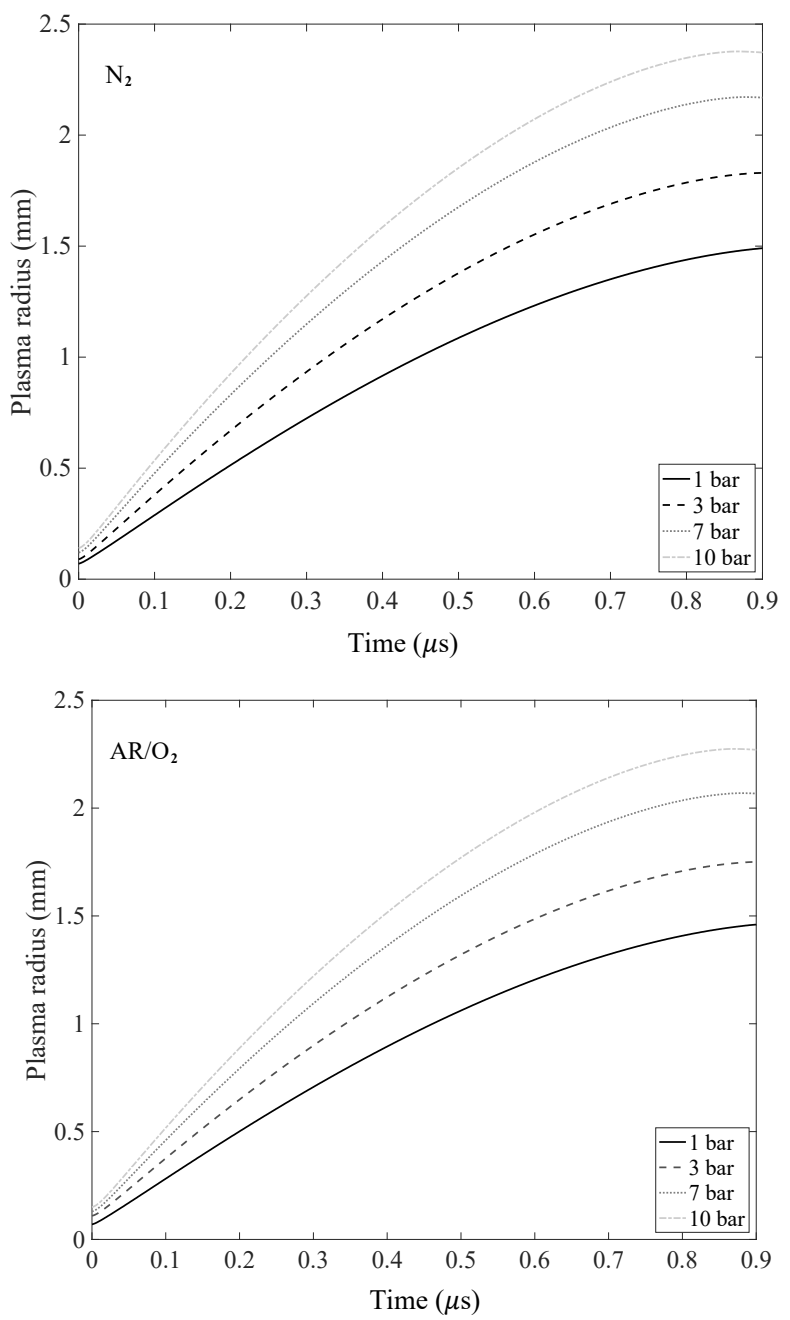

Fig. 4. Calculated time-dependent plasma channel radius for $\mathrm{N}_{2}$ and the $\mathrm{Ar} / \mathrm{O}_{2}$ mixture at different gas pressures.

Fig. 5 presents a cumulative figure for plasma radii in all tested gases, which demonstrates the time-dependent plasma radii as functions of the gas pressure at a specific time after breakdown, $900 \mathrm{~ns}$.

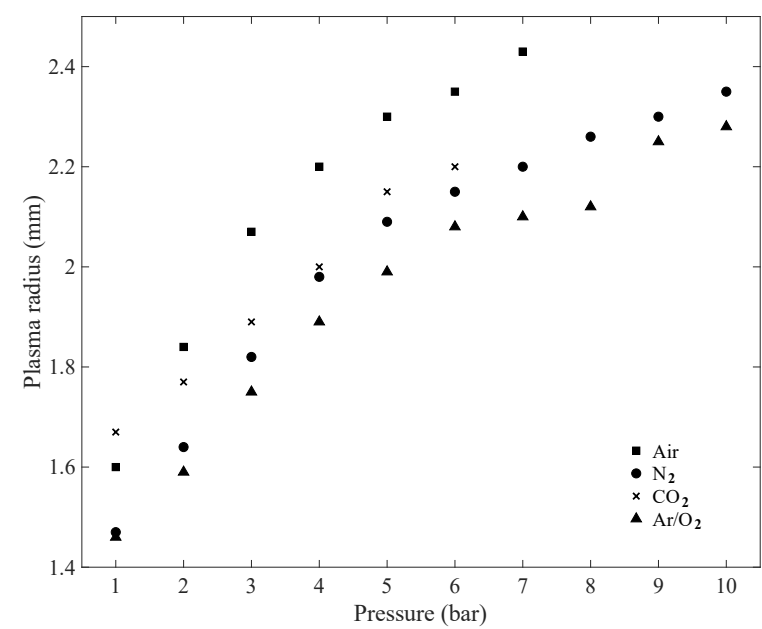

Fig. 5. Calculated plasma radius at $900 \mathrm{~ns}$ after breakdown as a function of the gas pressure, for all tested gases.

It can be seen that plasma radii increase with an increase in the gas pressure. This may be due to the higher breakdown voltages at higher pressures, resulting in more energy being delivered into the plasma channel, which will lead to greater Joule heating in the plasma and channel expansion. The calculated channel radii for air and $\mathrm{CO}_{2}$ behave similarly and they are not shown here.

At time $\sim 900 \mathrm{~ns}$, the analytically-obtained current reaches its first maximum in all gases, as the frequency of current oscillations is mainly dictated by the circuit capacitance and inductance in this model. In general, all gases show a linear increase in the time-dependent plasma radius as the gas pressure increases. As can be seen from Fig. 5, dry air has the largest radius of the plasma channel amongst all tested gases (at the same gas pressure), followed by $\mathrm{CO}_{2}$ and $\mathrm{N}_{2}$, while the $\mathrm{Ar} / \mathrm{O}_{2}$ mixture has the lowest radius under the same conditions. The calculated plasma channel radius shows a functional dependency on the gas pressure similar to the functional dependency of self-breakdown voltage on the gas pressure obtained in [21]. This can be explained by the higher energy delivered into the plasma channel at higher pressure in the PCS (higher breakdown voltage), resulting in the fast expansion of the plasma channel.

\section{Time-Dependent Resistance of the Plasma Channel}

The main focus of the present work is on the investigation of the time-dependent plasma resistance in the breakdown channel. The time-dependent plasma resistance has been obtained using three different approaches: I) by direct calculation of $R_{S}(t)$ using Kirchhoff's equation (5) and data from experimental current waveforms; II) using the Braginskii approach to calculate the transient radius of the plasma channel, which is then used to obtain the resistance of the cylindrical plasma channel by (1) - (3); III) using Kushner's approach (4), with experimental current waveforms and the radius of the channel obtained by the Braginskii equation (1).

Initial solutions of Kirchhoff's equation (5) produced a relatively "noisy" form for $R_{S}(t)$, due to noise in the 
experimental current wave-forms. To minimise this effect, current waveforms were pre-processed in MATLAB to reduce the noise content before their use in (5): the de-noising procedure included filtering the current waveforms using a fourth-order low-pass digital Butterworth filter.

Fig. 6 shows an example of the resistance of the plasma channel in air at 1 bar, obtained using all three methods. The transient resistance of the plasma channel obtained by (5) is shown in Fig. 6 as a solid line. The transient resistances obtained by both Braginskii's and Kushner's models for the same conditions are also shown in Fig. 6 as dot-dashed and dashed lines, respectively.
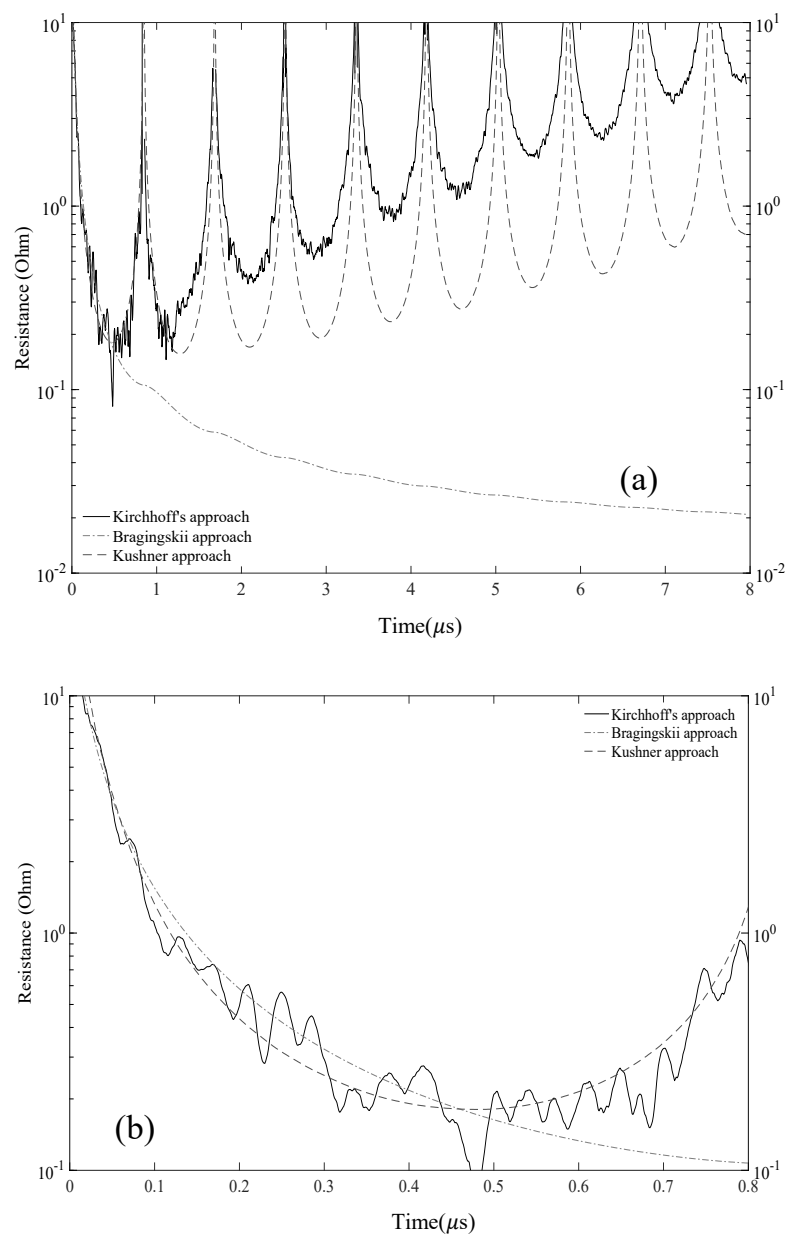

Fig. 6. The plasma channel resistance for air at 1 bar; (a) Calculated timedependent plasma resistances for breakdown channel during several current oscillations; (b) Zoomed-in view of plasma resistance obtained by different methods during the first half of current oscillation.

The plasma channel resistances obtained by all methods show a fast decay during the initial stage of the transient process, i.e. during the first quarter of the current oscillation. There is a good agreement between the Braginskii model, the Kushner model, and the data from solutions to Kirchhoff's equation. In Fig 6(b), it can be seen that the plasma resistance reduces to below $200 \mathrm{~m} \Omega$.

However, after this initial stage, the resistance predicted by the Braginskii model continues to fall, while the solution to Kirchhoff's equation indicates that the resistance of the channel starts to increase as the current flowing through the channel decreases. The Kushner model follows the behaviour of the Kirchhoff solution closely over the next half-period of the current oscillation, until the second minima in the channel resistance. After this time, the channel resistance from the Kushner model shows a similar periodicity to that obtained by solving Kirchhoff's equation, but predicts much lower values for the successive minima in the values of $R_{S}(t)$.

In many practical pulsed power applications, only the first quarter of the current oscillation is considered to be important. As can be seen from Fig. 6(b), all three approaches provide similar functional behaviour of the transient resistance during this time interval. This close correlation for the three approaches was observed for every gas, and at every tested pressure. The plasma resistance reaches its minimum value at the first quarter of the current oscillation, when the current in the spark channel reaches its maximum. The minimum values of the spark resistance were obtained for all investigated gases, for air, $\mathrm{N}_{2}, \mathrm{CO}_{2}$ and the $\mathrm{Ar} / \mathrm{O}_{2}$ mixture, using all three models. These minimum resistances were obtained as average values for 10 breakdown events, and are shown in Fig. 7 as functions of the gas pressure.

Inspection of the results provided in Fig. 7 demonstrates that there is no significant difference in the minimum values of the time-dependent resistance of spark channels in these gases. It was found that the minimum resistance of the spark channel for all investigated gases significantly decreases with an increase in the gas pressure in the PCS.

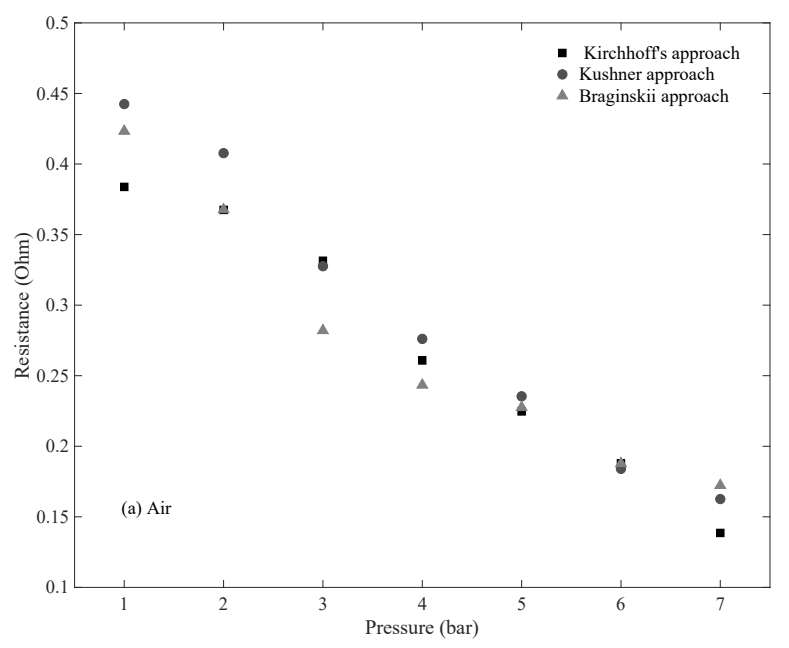



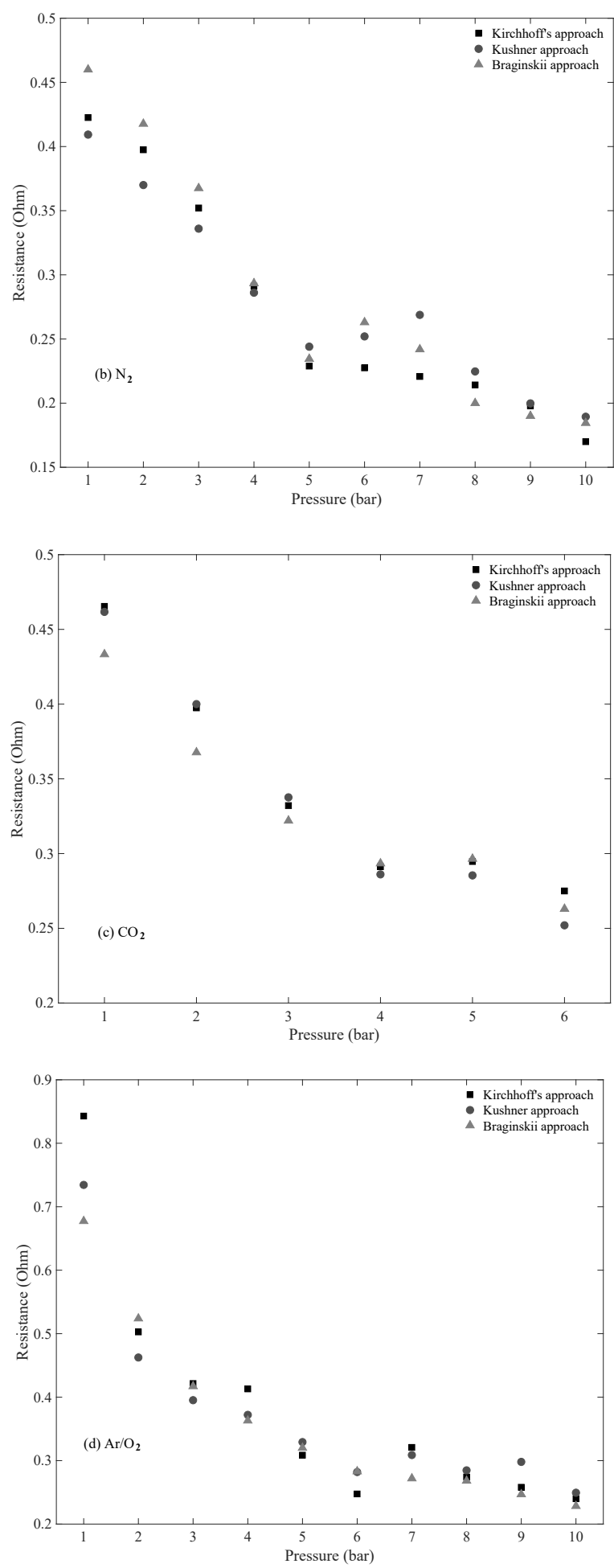

Figure 7. Minimum resistance at the first $1 / 4$ of the current oscillation for all investigated gases.

Fig. 8 shows the obtained minimum plasma resistance for all tested gases as a function of the peak current. Each point in this graph was calculated as the average value of the resistance obtained by all three methods. The minimum resistance decreases with an increase in the peak current in the postbreakdown circuit. As Fig. 9 shows, that there is no significant difference in the minimum resistance at the peak current for $\mathrm{N}_{2}$, and the $\mathrm{Ar} / \mathrm{O}_{2}$ mixture; the resistance in air is slightly higher than the resistance in these two gases, but the highest values for the plasma resistance as a function of current have been obtained in $\mathrm{CO}_{2}$. The higher values of plasma resistance for a given peak current implies that there is considerably more energy dissipation in breakdown channels formed in $\mathrm{CO}_{2}$ as compared with those formed in the other tested gases. This difference in the plasma resistance of $\mathrm{CO}_{2}$ becomes higher with increasing peak current; for the highest observed peak current of $8.5 \mathrm{kA}$, the plasma resistance is 2.5 -times higher than that in $\mathrm{N}_{2}$ and air.

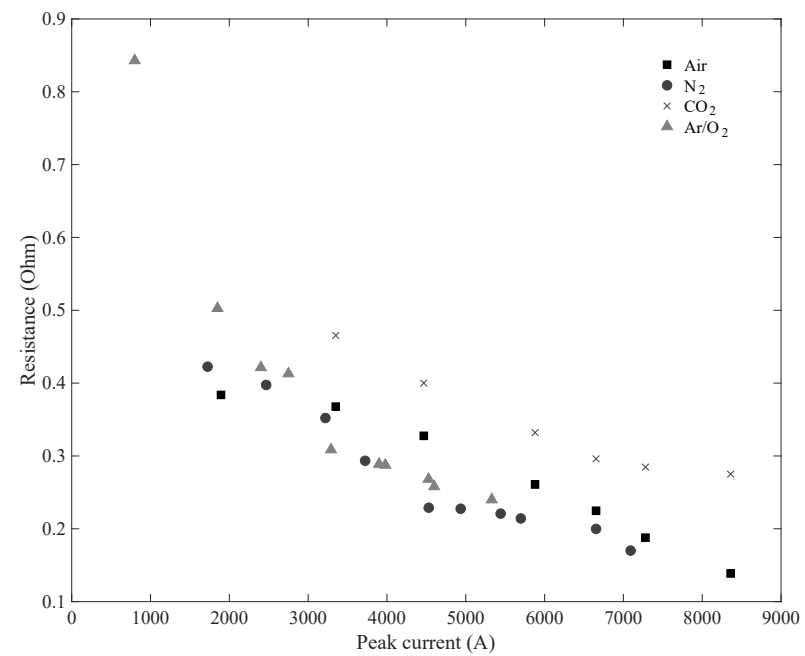

Fig. 8. The minimum plasma resistance of the spark channel as a function of the maximum post-breakdown current, for all tested gases.

\section{Energy Deposited in the Spark Channel}

The energy deposited into the plasma channel was obtained by integrating the product of the transient plasma resistance and the square of the current:

$$
E(t)=\int R_{S}(t) I(t)^{2} d t
$$

where $R_{S}(t)$ and $I(t)$ are the time-dependent resistance of, and current through, the plasma channel, respectively.

Fig. 9 shows an example of the energy deposited into the plasma channel in the case of a breakdown in dry air at 7 bar gauge. The $80 \mathrm{nF}$ energy-storage capacitance in the pulse driving circuit was charged to $32 \mathrm{kV}$, thus the total energy available in the discharge was $\sim 40 \mathrm{~J}$. The energy deposited into the plasma channel during the discharge reached $\sim 20 \mathrm{~J}$ at the end of the current oscillation process, which accounts for $\sim 50 \%$ of the energy stored in the capacitor. 


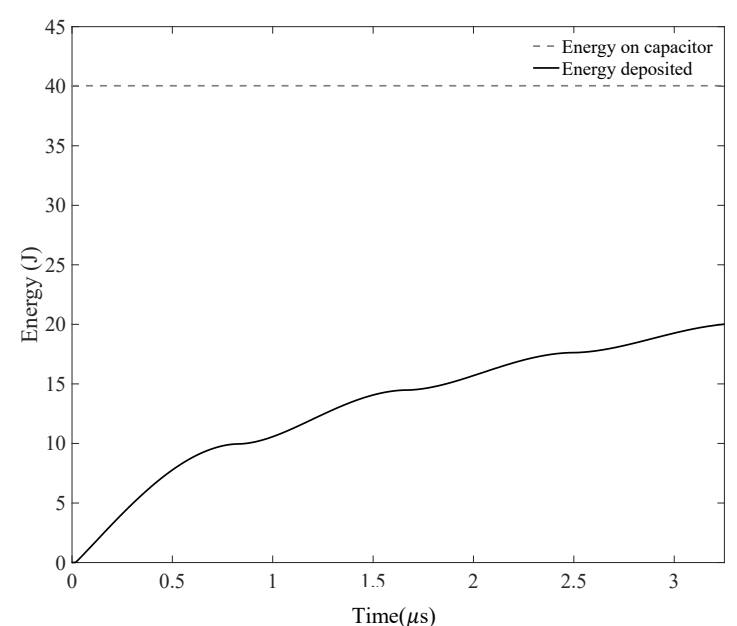

Fig. 9. Energy deposited in the spark channel in air at 7 bar.

The energy released in the spark channel is plotted in Fig. 10 as a function of the gas pressure in the PCS. This figure shows that the energy increases with an increase in the gas pressure due to higher plasma resistance at higher pressures. Also, it can be seen that the largest value of energy was released in the spark breakdown channel in $\mathrm{CO}_{2}$. The energy released in the plasma channel in air was also significantly higher than the energy released in the breakdown plasma channel in $\mathrm{N}_{2}$ and the $\mathrm{Ar} / \mathrm{O}_{2}$ mixture. This is due to higher breakdown voltages observed in the PCS filled with $\mathrm{CO}_{2}$ and air, with higher breakdown voltages resulting in higher energy available in the discharge.

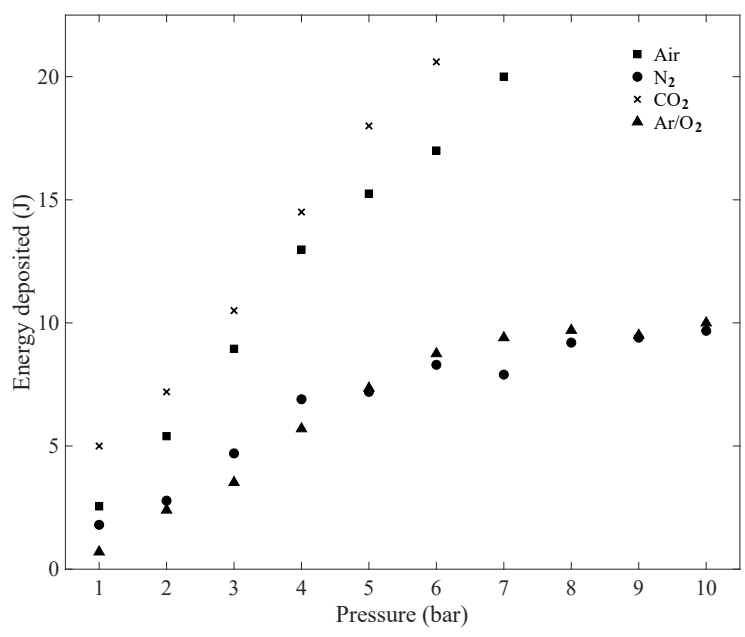

Figure 10. The maximum energy deposited in the spark channel for all tested gases as a function of gas pressure in the PCS.

\section{CONCLUSIONS}

In this paper, three different models have been used to study the dynamic resistance of the spark channel: the $R L C$ Kirchhoff's lumped-element circuit model, the Braginskii model, and the Kushner model. The $R L C$ model assumes that the post-breakdown circuit can be described as an $R L C$ lumpedelement equivalent circuit, with a time-dependent plasma

resistance. Thus, the plasma resistance can be obtained by solving Kirchhoff's equation. The Braginskii model assumes that the plasma channel expands hydrodynamically and takes into account the energy balance in a channel filled with plasma with a constant conductivity. The time-dependent radius of the plasma channels was obtained by the Braginskii model, and demonstrated good agreement with the channel radius observed in optical experiments, [35]. The Kushner model of the transient spark breakdown requires the current waveform and an analytical dynamic radius for the spark channel.

All three models were used to investigate the transient plasma resistance in the developed multi-electrode plasma closing switch, filled with different gases.

It was found that all three methods provide identical functional behaviour of the time-dependent plasma resistance during the first quarter-cycle of the current oscillation. At longer times, the Kushner model demonstrates a better fit to the resistance obtained by Kirchhoff's approach. Both Kirchhoff and Kushner approaches cannot predict the resistance value expected when the current passes through zero. The Braginskii model provides unrealistically low values of resistance after the first quarter-cycle of the current oscillation, and therefore cannot be used to determine the channel behaviour for underdamped current oscillations.

It was found that the minimum resistance of the postbreakdown plasma channel depends on the current through the channel and on the type of gas used in the PCS. The plasma resistance in $\mathrm{CO}_{2}$ was higher than the plasma resistance in the other gases at the same values of peak current. Also, it was established that the maximum energy deposited in the spark channel is highest for a $\mathrm{CO}_{2}$-filled switch. The energy deposited into the channel increases with an increase in the gas pressure, due to the higher plasma resistance at higher pressures.

The presented analysis will help in further understanding of the dynamic characteristics of gas-filled spark gap switches. It is expected that the analytical methods used in the present study can be deployed to optimise the parameters of plasma closing switches filled with environmentally-friendly and lower environmental impact gases, to improve their operational performance.

\section{REFERENCES}

J. R. Woodworth et al., "Low-inductance gas switches for linear transformer drivers," Physical Review Special Topics - Accelerators and Beams, vol. 12, no. 6, p. 060401, 06/08/ 2009, doi: 10.1103/PhysRevSTAB.12.060401.

M. E. Savage, "Final results from the high-current, high-action closing switch test program at Sandia National Laboratories," IEEE Transactions on Plasma Science, vol. 28, no. 5, pp. 1451-1455, 2000, doi: 10.1109/27.901213.

R. S. Clark, L. F. Rinehart, M. T. Buttram, and J. F. Aurand, "An Overview of Sandia National Laboratories' Plasma Switched, Gigawatt, Ultra-Wideband Impulse Transmitter Program," in UltraWideband, Short-Pulse Electromagnetics, H. L. Bertoni, L. Carin, and L. B. Felsen Eds. Boston, MA: Springer US, 1993, pp. 93-98. M. A. Malik, K. H. Schoenbach, T. M. Abdel-Fattah, R. Heller, and C. Jiang, "Low Cost Compact Nanosecond Pulsed Plasma System for Environmental and Biomedical Applications," Plasma Chemistry and Plasma Processing, vol. 37, no. 1, pp. 59-76, 2017/01/01 2017, doi: 10.1007/s11090-016-9747-9. 
[5] G. Schaefer, M. Kristiansen, and A. H. Guenther, Gas discharge closing switches. New York, N.Y.: Plenum Press (in English), 1990.

[6] T. P. Sorensen and V. M. Ristic, "Rise time and time-dependent spark-gap resistance in nitrogen and helium," Journal of Applied Physics, vol. 48, no. 1, pp. 114-117, 1977/01/01 1977, doi: $10.1063 / 1.323311$

[7] H. Wang, Q. Zhang, J. Wei, X. Liu, and A. Qiu, "Research on Erosion Property of Field-Distortion Gas Switch Electrode in Nanosecond Pulse," IEEE Transactions on Plasma Science, vol. 40, no. 6, pp. 1733-1742, 2012, doi: 10.1109/TPS.2012.2191162.

[8] A. R. Dick, S. J. MacGregor, M. T. Buttram, R. C. Pate, L. F. Rinehart, and K. R. Prestwich, "Breakdown phenomena in ultra-fast plasma closing switches," IEEE Transactions on Plasma Science, vol. 28, no. 5, pp. 1456-1462, 2000, doi: 10.1109/27.901214.

[9] W. K. Cary and J. A. Mazzie, "Time-resolved resistance during spark gap breakdown," IEEE Transactions on Electron Devices, vol. 26, no. 10, pp. 1422-1427, 1979, doi: 10.1109/T-ED.1979.19625.

[10] M. Toepler, "Zusammenhang zwischen Funken, Büschellichtbogen und Bogen," Archiv für Elektrotechnik, vol. 18, no. 6, pp. 563-566, 1927/11/01 1927, doi: 10.1007/BF01656201.

[11] R. Rompe and W. Weizel, "Über das Toeplersche Funkengesetz," Zeitschrift für Physik, vol. 122, no. 9, pp. 636-639, September 01 1944, doi: 10.1007/bf01330625.

[12] S. I. Braginskii, "Theory of the Development of a Spark Channel," Sov. Phys. JETP, vol. 34, pp. 1068-1074, 1958 1958. [Online]. Available: https://ci.nii.ac.jp/naid/10019124452/en/.

[13] M. J. Kushner, W. D. Kimura, and S. R. Byron, "Arc resistance of laser-triggered spark gaps," Journal of Applied Physics, vol. 58, no. 5, pp. 1744-1751, 1985, doi: 10.1063/1.336023.

[14] H. Akiyama, M. Kristiansen, H. Krompholz, and B. Maas, "Currentvoltage characteristics of a high-current pulsed discharge in air," IEEE Transactions on Plasma Science, vol. 16, no. 2, pp. 312-316, 1988, doi: 10.1109/27.3830.

[15] K. Takaki and H. Akiyama, "Resistance of pulsed arc discharge in air and SF/sub 6," in PPPS-2001 Pulsed Power Plasma Science 2001. 28th IEEE International Conference on Plasma Science and 13th IEEE International Pulsed Power Conference. Digest of Papers (Cat. No.01CH37251), 17-22 June 2001 2001, vol. 2, pp. 1758-1761 vol.2, doi: 10.1109/PPPS.2001.1001912.

[16] T. G. Engel, A. L. Donaldson, and M. Kristiansen, "The pulsed discharge arc resistance and its functional behavior," IEEE Transactions on Plasma Science, vol. 17, no. 2, pp. 323-329, 1989, doi: $10.1109 / 27.24643$.

[17] W. D. Greason, "Methodology to study the resistance of spark discharges," IEEE Transactions on Industry Applications, vol. 35, no. 2, pp. 359-365, 1999, doi: 10.1109/28.753629.

[18] J. P. VanDevender, "The resistive phase of a high-voltage water spark," Journal of Applied Physics, vol. 49, no. 5, pp. 2616-2620, 1978/05/01 1978, doi: 10.1063/1.325205.

[19] B. Lin and Q. Chow, "Electric characteristics of pseudo-spark during breakdown phase," Journal of Physics D: Applied Physics, vol. 26, no. 7, pp. 1057-1060, 1993/07/14 1993, doi: 10.1088/0022$3727 / 26 / 7 / 007$.

[20] R. T. Robiscoe, A. Kadish, and W. B. Maier, "Overdamped arc discharge data and an AWA model," IEEE Transactions on Plasma Science, vol. 19, no. 3, pp. 529-534, 1991, doi: 10.1109/27.87234.

[21] Y. Yao, I. V. Timoshkin, S. J. MacGregor, M. P. Wilson, M. J. Given, and T. Wang, "Breakdown Characteristics of Plasma Closing Switch Filled With Air, $\mathrm{N}_{2}, \mathrm{CO}_{2}$, and $\mathrm{Ar} / \mathrm{O}_{2}$," IEEE Transactions on Plasma Science, vol. 46, no. 10, pp. 3574-3583, 2018, doi: 10.1109/TPS.2018.2856306.

[22] A. E. Vlastós, R. Rompe, and W. Weizel, "The Resistance of Sparks," Journal of Applied Physics, vol. 43, no. 4, pp. 1987-1989, 1972, doi: 10.1063/1.1661429.

[23] P. Castera, "Energy coupling mechanisms in pulsed surface discharges for flow control," Ecole Centrale Paris, 2015ECAP0041, 2015. [Online]. Available: https://tel.archives-ouvertes.fr/tel01225674

[24] F. W. Grover, Inductance Calculations: Working Formulas and Tables (Dover phoenix editions). Dover Publications, 2004.

[25] T. W. Hussey, K. J. Davis, J. M. Lehr, N. F. Roderick, R. C. Pate, and E. Kunhardt, "Dynamics of nanosecond spark-gap channels," in Digest of Technical Papers. 12th IEEE International Pulsed Power
Conference. (Cat. No.99CH36358), 27-30 June 1999 1999, vol. 2, pp. 1171-1174 vol.2, doi: 10.1109/PPC.1999.823730.

[26] L. Spitzer, Physics of Fully Ionized Gases (Dover Books on Physics). Dover Publications, 2006.

[27] M. Janda, V. Martišovitš, and Z. Machala, "Transient spark: a dcdriven repetitively pulsed discharge and its control by electric circuit parameters," Plasma Sources Science and Technology, vol. 20, no. 3 , p.035015, 2011/04/19 2011, doi: 10.1088/09630252/20/3/035015.

[28] T. Shao et al., "Spark discharge formation in an inhomogeneous electric field under conditions of runaway electron generation," Journal of Applied Physics, vol. 111, no. 2, p. 023304, 2012/01/15 2012, doi: 10.1063/1.3677951.

[29] S. Heijkers, L. M. Martini, G. Dilecce, P. Tosi, and A. Bogaerts, "Nanosecond Pulsed Discharge for CO2 Conversion: Kinetic Modeling To Elucidate the Chemistry and Improve the Performance," The Journal of Physical Chemistry C, vol. 123, no. 19, pp. 12104-12116, 2019/05/16 2019, doi: 10.1021/acs.jpcc.9b01543.

[30] A. Kohut, G. Galbács, Z. Márton, and Z. Geretovszky, "Characterization of a copper spark discharge plasma in argon atmosphere used for nanoparticle generation," Plasma Sources Science and Technology, vol. 26, no. 4, p. 045001, 2017/03/01 2017, doi: 10.1088/1361-6595/aa5c2b.

[31] R. Montanomontano, M. Becerra, V. Cooray, M. Rahman, and P. Liyanage, "Resistance of Spark Channels," IEEE Transactions on Plasma Science, vol. 34, no. 5, pp. 1610-1619, 2006, doi: 10.1109/TPS.2006.883350.

[32] F. Santamaria and F. Román, "Experimental Study of a Submillimeter Spark-Gap," IEEE Transactions on Plasma Science, vol. 41, no. 4, pp. 985-992, 2013, doi: 10.1109/TPS.2013.2249531.

[33] E. Bazelyan, Y. Raizer, "Spark discharge", CRC press, 1997
[34] X. Li, X. Liu, F. Zeng, H. Yang, and Q. Zhang, "Study on Resistance and Energy Deposition of Spark Channel Under the Oscillatory Current Pulse," IEEE Transactions on Plasma Science, vol. 42, no. 9, pp. 2259-2265, 2014, doi: 10.1109/TPS.2014.2331346.

[35] P. Castera and P. Elias, "Resistance Models Applied to the Return Stroke Phase of Negative Pulsed Surface Discharges in Air," IEEE Transactions on Plasma Science, vol. 42, no. 7, pp. 1922-1931, 2014, doi: 10.1109/TPS.2014.2324637. IEEE Transactions on Plasma Science.

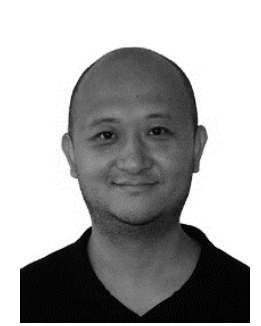

Yuan Yao was born in Beijing, China. He received the B.Eng. degree in Electronic and Electrical engineering from Beijing Institute of Technology, China, in 2003, and the M.Eng. degree from the Chinese Academy of Sciences (CAS) in 2008. He worked as an Engineer at the Institute of High Energy Physics, CAS, from 2003 to 2016.

Currently he is pursuing his Ph.D. degree in High Voltage Technology at the Department of Electronic and Electrical Engineering, University of Strathclyde, Glasgow. His current research interests include electrical breakdown in environmentally friendly gases, plasma closing switches, and corona discharges.

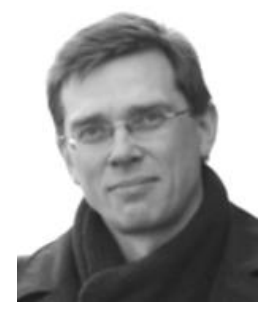

Igor V. Timoshkin (M'07-SM'14) received the degree in physics from Moscow State University, Moscow, Russia, in 1992, and the Ph.D. degree from the Imperial College of Science, Technology and Medicine (ICSTM), London, U.K., in 2001.

He was a Researcher at Moscow State Agro-Engineering University, Moscow, and then at the Institute for High Temperatures of Russian Academy of 
Sciences, Moscow. In 1997 he joined ICSTM. Then he joined the Department of Electronic and Electrical Engineering, University of Strathclyde, Glasgow, U.K., in 2001, where he became a Reader in 2016. His current research interests include pulsed power, transient spark discharges, environmental applications of non-thermal plasma discharges.

Dr. Timoshkin is a Voting Member of the Pulsed Power Science and Technology Committee in the IEEE Nuclear and Plasma Science Society; a member of International Advisory Committee of the IEEE Conference on Dielectric Liquids and a member of the International Scientific Committee of the Gas Discharges and Their Application Conference.

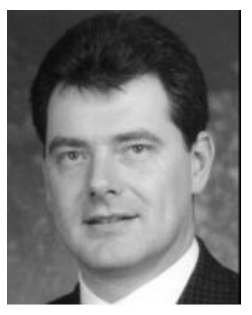

Scott J. MacGregor (M'95-SM'14) received the B.Sc. and Ph.D. degrees from the University of Strathclyde, Glasgow, U.K., in 1982 and 1986, respectively.

He was a Pulsed-Power Research Fellow in 1986 and a Lecturer in pulsed-power technology in 1989. In 1994, he became a Senior Lecturer, with a promotion to a Reader and a Professor of High Voltage Engineering, in 1999 and 2001, respectively. In 2006 and 2010, he became the Head of the Department of Electronic and Electrical Engineering and the Executive Dean of the Faculty of Engineering and has been the Vice-Principal with the University of Strathclyde, since 2014. His current research interests include high-voltage pulse generation, high-frequency diagnostics, high-power repetitive switching, high-speed switching, electronic methods for food pasteurization and sterilization, the generation of high-power ultrasound (HPU), plasma channel drilling, pulsed-plasma cleaning of pipes, and the stimulation of oil wells with HPU. Prof. MacGregor was a recipient of the 2013 IEEE Peter Haas Award. He was an Associated Editor of the IEEE Transitions On Dielectrics And Electrical Insulation in 2015.

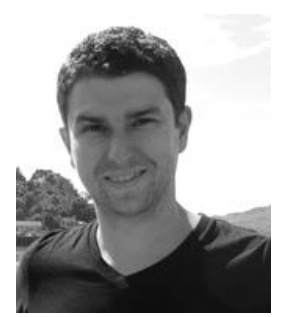

Mark P. Wilson (M'10) was born in Stranraer, Scotland, in 1982. He received the B.Eng. (with honours), M.Phil., and $\mathrm{Ph} . \mathrm{D}$. degrees in electronic and electrical engineering from the University of Strathclyde, Glasgow, U.K., in 2004, 2007, and 2011, respectively. $\mathrm{He}$ is presently based in the High Voltage Technologies research group at the University of Strathclyde, where his research interests include interfacial surface flashover, nanodielectrics, and the practical applications of high-power ultrasound, corona discharges, and pulsed electric fields. Mark is a member of the IEEE Nuclear and Plasma Sciences Society, from whom he received a Graduate Scholarship Award in 2011, the IEEE Dielectrics and Electrical Insulation Society, and the IET.

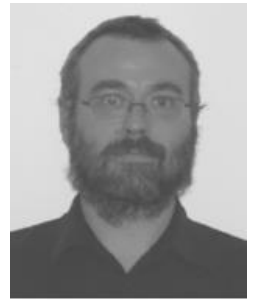

Martin J. Given (M'99-SM'11) received the B.Sc. degree in physics from the University of Sussex, Brighton, U.K., in 1981, and the Ph.D. degree in electronic and electrical engineering from the University of Strathclyde, Glasgow, U.K., in 1996.

He is currently a Senior Lecturer with the Department of Electronic and Electrical Engineering, University of Strathclyde. His current research interests include aging processes and condition monitoring in solid and liquid insulation systems, high-speed switching, and pulse power.

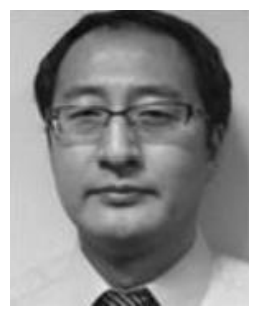

Tao Wang received the B.Eng. and M.Sc. degrees from Northeast China Dianli University, Jilin, China, in 1993 and 1996, respectively, and the Ph.D. degree from the University of Strathclyde, Glasgow, U.K., in 2005.

He joined the Newland Entech, China, as a Research Fellow, developing highefficiency industrial ozone generator. $\mathrm{He}$ joined the Department of Electronic and Electrical Engineering, University of Strathclyde, as a Lecturer, in 2010. His current research interests include nonthermal gas discharges and their applications in gas synthesis, water disinfection, and advanced oxidation process in water. 